\title{
Syntaxin 1B Suppresses Macropinocytosis and Semaphorin 3A-Induced Growth Cone Collapse
}

\author{
Hiroyuki Kabayama, ${ }^{1,2}$ Makoto Takeuchi, ${ }^{1}$ Masahiko Taniguchi, ${ }^{3}$ Naoko Tokushige, ${ }^{1}$ Shunji Kozaki, ${ }^{4}$ Akihiro Mizutani, ${ }^{1}$ \\ Takeshi Nakamura, ${ }^{2,5}$ and Katsuhiko Mikoshiba ${ }^{1,2}$ \\ ${ }^{1}$ Laboratory for Developmental Neurobiology, Brain Science Institute, The Institute of Physical and Chemical Research, Wako, Saitama 351-0198, Japan, \\ ${ }^{2}$ International Cooperative Research Project-Solution-Oriented Research for Science and Technology, Japan Science and Technology Agency, Kawaguchi, \\ Saitama 332-0012, Japan, ${ }^{3}$ Department of Biochemistry, Cancer Research Institute, Sapporo Medical University School of Medicine, Chuo-ku, Sapporo \\ 060-8556, Japan, ${ }^{4}$ Laboratory of Veterinary Epidemiology, Department of Veterinary Science, Graduate School of Life and Environmental Sciences, Osaka \\ Prefecture University, Sakai-shi, Osaka 599-8531, Japan, and 5Department of Physiology, Juntendo University Faculty of Medicine, Bunkyo-ku, Tokyo \\ 113-8421, Japan
}

Growth cone collapse is a crucial process for repulsive axon guidance and is accompanied by a reduction in growth cone surface area. This process of reduction may be regulated by endocytosis; however, its molecular mechanism is unclear. Macropinocytosis is a clathrinindependent form of endocytosis in which large areas of plasma membrane can be engulfed. We have reported previously that macropinocytosis is induced in growth cones of chick dorsal root ganglion neurons by semaphorin $3 \mathrm{~A}$ (Sema3A), a repulsive axon guidance cue, and that Sema3A-induced reduction in growth cone surface area and macropinocytic vacuole area were correlated, suggesting a positive role for macropinocytosis in Sema3A-induced growth cone collapse. In the present study, we found that syntaxin 1B (Syx1B), a membrane trafficking protein, is a negative regulator of macropinocytosis, and its expression is downregulated by Sema3A signaling. Macropinocytosis inhibitor ethylisopropylamiloride or Syx1B overexpression suppressed Sema3A-induced macropinocytosis and growth cone collapse. These results indicate that Syx1B couples macropinocytosis-mediated massive internalization of the plasma membrane to Sema3A-induced growth cone collapse.

\section{Introduction}

Macropinocytosis is a clathrin-independent endocytosis leading to the formation of large endocytic vacuoles (called macropinosomes) and is generated by two distinct processes: actin-driven circular membrane ruffling and fusion of membrane ruffles to the plasma membrane (Swanson and Watts, 1995). Macropinosomes are heterogeneous in size and range from 0.2 to $5 \mu \mathrm{m}$ in diameter, whereas micropinosomes are smaller than $0.2 \mu \mathrm{m}$ (Swanson and Watts, 1995; Johannes and Lamaze, 2002; EngqvistGoldstein and Drubin, 2003). Macropinosomes can be visualized by the incorporation of high-molecular-weight $(>10 \mathrm{kDa}) \mathrm{dex}-$ tran (Swanson and Watts, 1995). In various cells, macropinocytosis is usually involved in the nonselective bulk uptake of membrane components and/or fluids. Macropinocytosis also

\footnotetext{
Received May 20, 2010; revised March 26, 2011; accepted March 28, 2011.

Author contributions: H.K. and K.M. designed research; H.K., M.Tak., M.Tan., N.T., S.K., A.M., and T.N. performed research; H.K. analyzed data; H.K. wrote the paper.

This study is supported by grants from International Cooperative Research Project-Solution-Oriented Research for Science and Technology (K.M.) and in part by a Grant-in-Aid for Young Scientists (B) (H.K.) from the Japanese Ministry of Education, Culture, Sports, Science, and Technology. We thank Drs. Fumiaki Hamazato and Hirohide Iwasaki for valuable discussions and critical comments. We are grateful to the Support Unit for Bio-material Analysis, The Institute of Physical and Chemical Research Brain Science Institute Research Resources Center, for help with DNA sequencing analysis.

Correspondence should be addressed to either Dr. Katsuhiko Mikoshiba or Dr. Hiroyuki Kabayama, Laboratory for Developmental Neurobiology, Brain Science Institute, The Institute of Physical and Chemical Research, 2-1 Hirosawa, Wako-shi, Saitama 351-0198, Japan. E-mail: mikosiba@brain.riken.jp or kabayama@brain.riken.jp.

DOI:10.1523/JNEUROSCI.2718-10.2011

Copyright $\odot 2011$ the authors $\quad 0270-6474 / 11 / 317357-08 \$ 15.00 / 0$
}

plays a key role in the uptake of some bacteria and viruses (Mercer and Helenius, 2009), cell-penetrating peptides (Wadia et al., 2004), or prion (Wadia et al., 2008).

Semaphorin 3A (Sema3A) is a secreted repulsive axon guidance cue (Luo et al., 1993; Püschel et al., 1995; Taniguchi et al., 1997, 2003; Taniguchi and Shimizu, 2004) inducing growth cone collapse, which is defined as a reduction in the cell surface area of growth cones (Mikule et al., 2002). One possible mechanism of reduction is clathrin-mediated endocytosis of the plasma membrane. In fact, clathrin-mediated endocytosis of a Sema3A receptor (neuropilin-1) is induced by low-dose Sema3A (Piper et al., 2005); however, this type of endocytosis prevents growth cone collapse by high-dose Sema3A. Most importantly, high-dose Sema3A can induce growth cone collapse when clathrin-mediated endocytosis of receptors is inhibited (Piper et al., 2005). These findings suggest that clathrin-mediated endocytosis of receptors may not be the mechanism involved in Sema3A-mediated reduction in the cell surface area of growth cones; rather, it plays a negative role in Sema3A-induced growth cone collapse. Sema3A is also known to induce endocytic large vacuoles and the subsequent retrograde membrane trafficking during growth cone collapse (Fournier et al., 2000; Jurney et al., 2002). We had demonstrated previously that these large endocytic vacuoles are formed by macropinocytosis and that there is the inverse correlation between the area of Sema3Ainduced macropinosomes and the cell surface area of growth cones during growth cone collapse of dorsal root ganglion (DRG) neurons (Kabayama et al., 2009), suggesting the important positive role of 
macropinocytosis-mediated massive membrane retrieval in Sema3A-induced reduction in the cell surface area of growth cones.

Neurotoxin C1 is a zinc-dependent metalloprotease that cleaves in vivo syntaxin isoforms 1-3, which are involved in vesicle membrane trafficking (Schiavo et al., 1995). A previous study showed that neurotoxin $\mathrm{C} 1$ induces a reduction in the growth cone area (growth cone collapse), the formation of macropinosome-like large vacuoles, and the subsequent retrograde transport of vacuoles in growth cones by the cleavage of syntaxin 1 (Igarashi et al., 1996). In this study, we found that neurotoxin $\mathrm{C} 1$-induced vacuoles are formed by macropinocytosis and that syntaxin 1B (Syx1B) is a negative regulator of macropinocytosis. Consequently, we investigated the involvement of Syx1Bregulated macropinocytosis in Sema3Ainduced growth cone collapse.

\section{Materials and Methods}

Materials. F-12 medium (Invitrogen) or L-15 medium was used as the culture medium. Alexa Fluor 488-labeled high-molecular-weight $(10 \mathrm{kDa})$ dextran (Alexa Fluor 488-dextran) stock solution (Invitrogen) was prepared in F-12 medium with or without neurotoxin C1. Anti-Syx1B antibody, purchased from Synaptic Systems, was generated against a synthetic peptide (GKLAIFTDDIKMDSQMT) corresponding to amino acid residues 171-187 in mouse Syx1B. This peptide is identical to that present in chick Syx1B cloned in this study. Epoxomicin was purchased from Peptide Institute and used at a final concentration of $1 \mu \mathrm{M}$. Neurotoxin C1 was purified from Clostridium botulinum type C (Kurazono et al., 1985) and used at a final concentration of $200 \mathrm{~nm}$.

Cell culture. Primary cultures were prepared from embryonic day 7-9 male and female chick (Gallus gallus) embryos as described previously (Kabayama et al., 1999). All experimental protocols were approved by the RIKEN Institutional Animal Care and Use Committee. DRG neurons were cultured in F-12 medium supplemented with $50 \mathrm{ng} / \mathrm{ml}$ nerve growth factor (NGF) (Calbiochem) or in L-15 medium (Invitrogen) supplemented with $10 \%$ fetal bovine serum, $2 \mathrm{~mm}$ glutamine, $5 \mathrm{ng} / \mathrm{ml} \mathrm{NGF,} \mathrm{0.6 \%}$ glucose, and gentamicin (Roche Diagnostics). After electroporation, the cells were cultured in an F-12-based medium supplemented with $1 \times$ MACS Supplement B27 PLUS (Miltenyi Biotec) or an L-15-based medium supplemented with $1 \times$ MACS Supplement B27 PLUS .

Endocytosis of Alexa Fluor 488-dextran. To visualize the endocytic activity in growth cones, cells were exposed to 0.5 $\mathrm{mg} / \mathrm{ml}\left(M_{\mathrm{r}} 10,000\right)$ fixable Alexa Fluor 488 -dextran (Invitrogen) for 90 min with $200 \mathrm{~nm}$ neurotoxin C1. Images of the living growth cones were obtained after washing three times with Alexa Fluor 488-dextrandeficient balanced salt saline (BSS) imaging buffer, $\mathrm{pH} 7.4$, comprising the following (in $\mathrm{mM}$ ): $2 \mathrm{CaCl}_{2}, 5.4 \mathrm{KCl}, 115 \mathrm{NaCl}, 10$ glucose, and 20 HEPES. To quantify the percentage of dextran-labeled growth cones, the cells were cultured with F-12-based medium and treated with neurotoxin $\mathrm{C} 1$ in the presence of Alexa Fluor 488-dextran for $90 \mathrm{~min}$, washed three
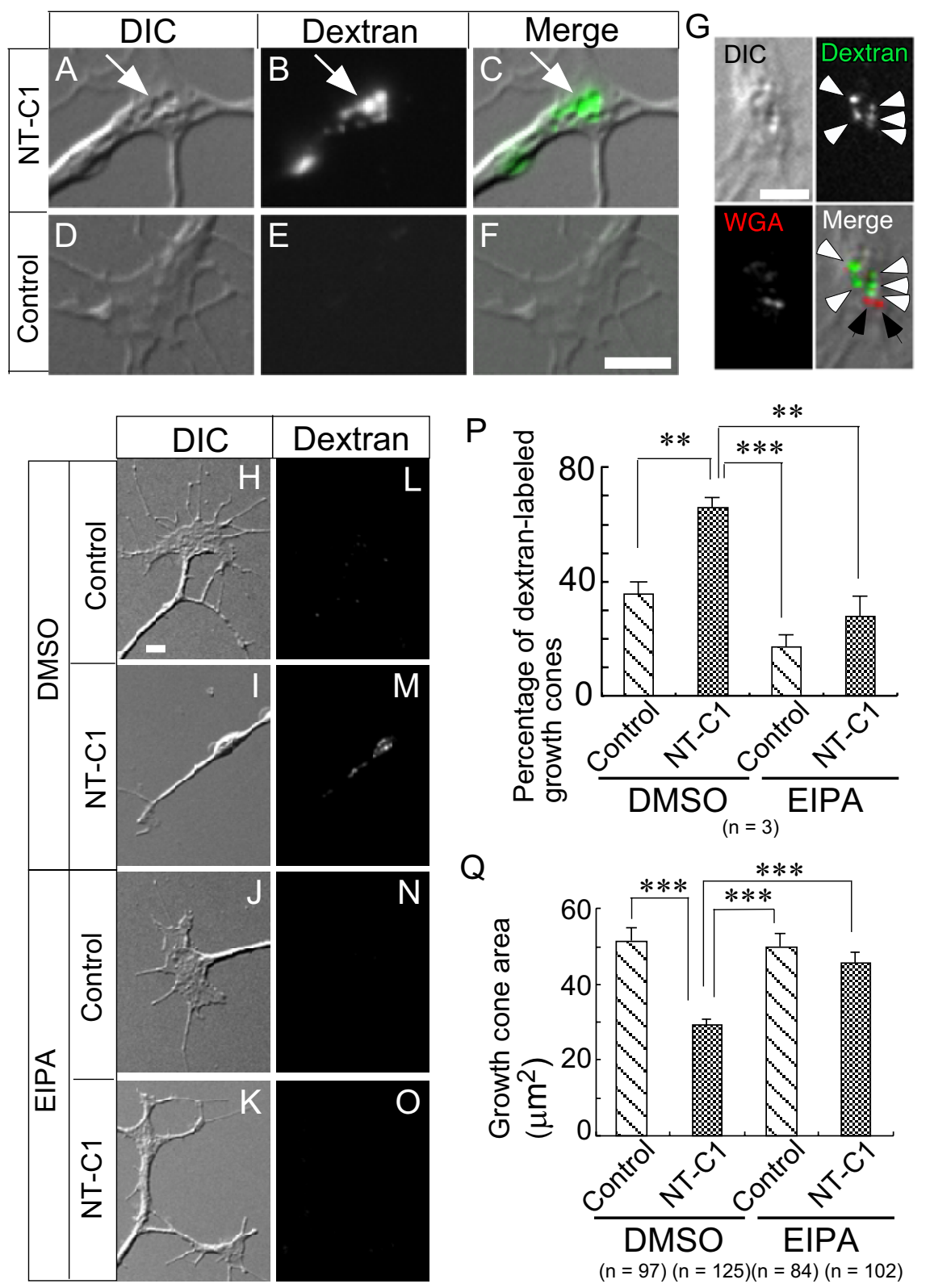

Figure 1. Neurotoxin (1-induced vacuoles are formed by macropinocytosis. $\boldsymbol{A}-\boldsymbol{F}, \mathrm{DIC}$, fluorescence, and merged images of a living growth cone treated with neurotoxin $\mathrm{C} 1(\mathrm{NT}-\mathrm{C} 1 ; \boldsymbol{A}-\boldsymbol{C})$ or a control buffer $(\boldsymbol{D}-\boldsymbol{F})$ in the presence of Alexa Fluor $488-$ dextran. The arrows indicate large vacuoles colocalized with Alexa Fluor 488-dextran. G, WGA-labeled GCVs were not colocalized with neurotoxin (1-induced large vacuoles. DIC image, fluorescence images of Alexa Fluor 488-dextran (green) and Alexa Fluor 594 -WGA (red), and the merged image of a living growth cone treated with neurotoxin C1. The white arrowheads indicate Alexa Fluor 488 - dextran localized to large vacuoles. The black arrows indicate Alexa Fluor 594-WGA fluorescent puncta. $\boldsymbol{H - O}, \mathrm{DIC}$ and fluorescence images of a fixed growth cone treated with a control buffer $(\boldsymbol{H}, \boldsymbol{L}, \boldsymbol{J}, \boldsymbol{N})$ or neurotoxin $(1(\boldsymbol{I}, \boldsymbol{M}, \boldsymbol{K}, \mathbf{0})$ in the presence of $\operatorname{DMSO}(\boldsymbol{H}, \boldsymbol{I}, \boldsymbol{L}, \boldsymbol{M})$ or $100 \mu \mathrm{m}$ EIPA $(\boldsymbol{J}, \boldsymbol{N}, \boldsymbol{K}, \mathbf{0}) . \boldsymbol{P}$, The percentage of growth cones labeled with Alexa Fluor 594 - dextran during 90 min neurotoxin C1 or control buffer treatment in the presence of DMSO or $100 \mu \mathrm{M}$ EIPA. ${ }^{* *} p<0.01,{ }^{* * *} p<0.001$, two-tailed non-repeated-measures ANOVA followed by Newman-Keuls post hoc multiple comparison test. $\mathbf{Q}$, Growth cone area treated with neurotoxin C1 or control buffer in the presence of DMSO or $100 \mu \mathrm{m}$ EIPA. ${ }^{* *} p<0.01$, two-tailed non-repeated-measures ANOVA followed by Newman-Keuls post hoc multiple comparison test. Error bars (in $\boldsymbol{P}$ and $\mathbf{Q}$ ) indicate SEM. Scale bar, $5 \mu \mathrm{m}$.

times with the Alexa Fluor 488-dextran-deficient F-12 medium, and immediately fixed with $4 \%$ PFA. In the experiments using siRNA, the cells transfected with siRNA were cultured with L-15-based medium as described previously (Kabayama et al., 1999) and exposed to $0.5 \mathrm{mg} / \mathrm{ml}$ $\left(M_{\mathrm{r}} 10,000\right)$ fixable Alexa Fluor 594-dextran (Invitrogen) for $30 \mathrm{~min}$ at $6 \mathrm{~h}$ after electroporation. The cells were fixed after washing with Alexa Fluor 594-dextran-deficient L-15-based medium. In the experiments using Sema3A, cells were cultured with F-12-based medium and exposed to $0.5 \mathrm{mg} / \mathrm{ml}$ Alexa Fluor 594 -dextran for $10 \mathrm{~min}$ in the presence of 1 

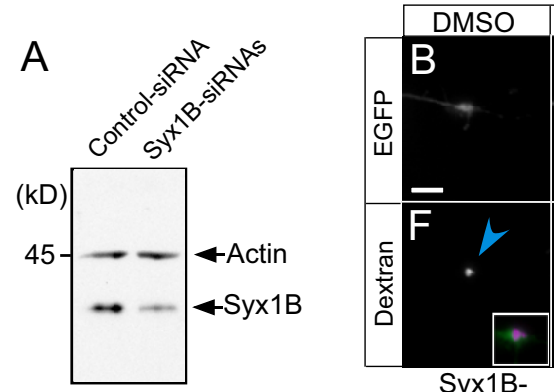

SiRNA

$+$

EGFP

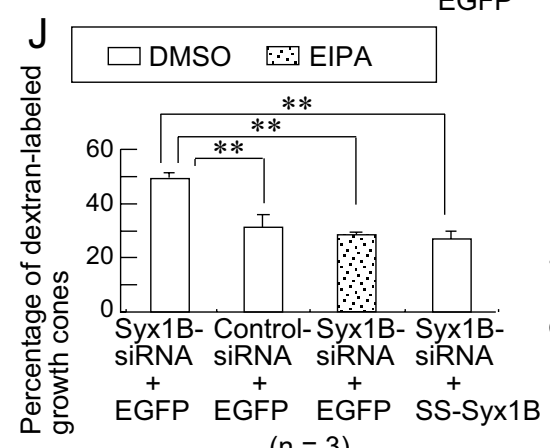

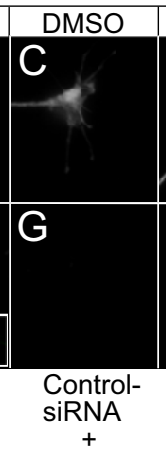

EIPA

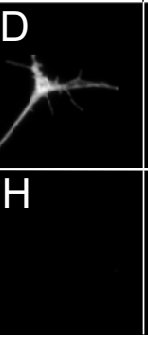

EGFP

DMSO

E

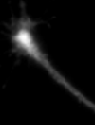

K $\square$ DMSO $\because$ EIPA

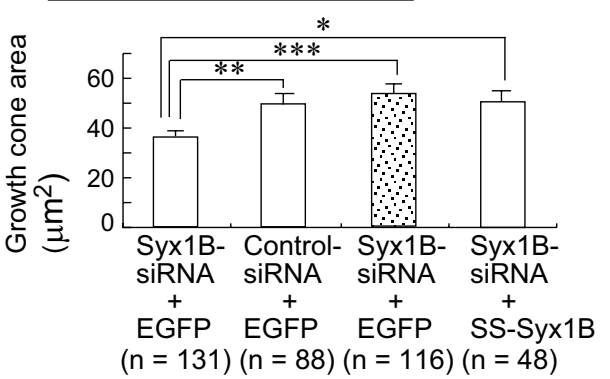
$(n=3)$

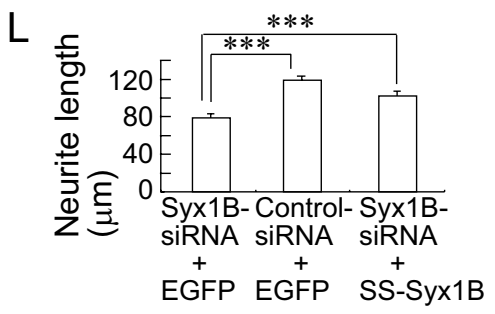

$(n=168)(n=137)(n=160)$

Figure 2. Syx $1 B$ is a negative regulator of macropinocytosis in growth cones. $A$, Western blot analysis of endogenous Syx $1 B$ expression level in DRG neurons transfected with Syx1B-siRNA or control siRNA. $\boldsymbol{B}$-I, Fluorescence EGFP (top, white) and Alexa Fluor 594 - dextran (bottom, white) images of a fixed growth cone cotransfected with the indicated siRNA and EGFP or EGFPlabeled SS-Syx1B in the presence of DMSO or $10 \mu \mathrm{m}$ EIPA. The blue arrowheads indicate incorporated Alexa Fluor 594 - dextran. The inset in $\boldsymbol{F}$ is an enlargement of merged image of EGFP ( $\boldsymbol{B}$, green) and dextran ( $\boldsymbol{F}$, magenta). $\boldsymbol{J}$, The percentage of siRNAtransfected growth cones labeled with Alexa Fluor 594 - dextran under the various conditions shown in $\boldsymbol{B}-\boldsymbol{I}$. $\boldsymbol{K}$, Growth cone area of siRNA-transfected growth cones shown in $\boldsymbol{B}-\boldsymbol{I}$. $\boldsymbol{L}$, Neurite length of siRNA-transfected cells shown in $\boldsymbol{B}, \boldsymbol{C}$, and $\boldsymbol{E}$. ${ }^{*} p<0.05$, ${ }^{* *} p<0.01,{ }^{* * *} p<0.001$, two-tailed non-repeated-measures ANOVA followed by Newman-Keuls posthoc multiple comparison test. Error bars (in $\boldsymbol{J}-\boldsymbol{L}$ ) indicate SEM. Scale bars, $10 \mu \mathrm{m}$.

$\mu \mathrm{g} / \mathrm{ml}$ Sema3A. The effects of macropinocytosis inhibitor ethylisopropylamiloride (EIPA) on dextran uptake, growth cone collapse, and neurite outgrowth were assayed by pretreating DRG neurons for 15 min with 10 or $100 \mu \mathrm{M}$ EIPA before addition of dextran. Fluorescence images of Alexa Fluor 488 and Alexa Fluor 594 in the growth cone area were obtained by GFP optics and Discosoma red (DsRed) optics, respectively. The percentage of growth cones that took up dextran was calculated from a random selection in the culture. In the experiments using siRNA, cells were cotransfected with EGFP-expressing vector for visualization of siRNA. The percentage of dextran-labeled growth cones was calculated from all EGFP-positive growth cones in the culture.

Labeling of growth cone vesicles. To visualize the growth cone vesicles (GCVs), the cells were exposed to $5 \mu \mathrm{g} / \mathrm{ml}$ Alexa Fluor 594-labeled wheat-germ agglutinin (WGA) (Alexa Fluor 594-WGA) (Invitrogen) for $10 \mathrm{~min}$ at $37^{\circ} \mathrm{C}$. Subsequently, the cells were washed three times with a prewarmed fresh medium without Alexa Fluor 594-WGA and cultured again. These cells were used for the experiments involving treatment with neurotoxin $\mathrm{C} 1$ and Alexa Fluor 488 -dextran at $60 \mathrm{~min}$ after washing.

Construction of expression vectors. Full-length chick Syx1B was obtained from an embryonic day 9 chick DRG cDNA library by PCR. The primers used were 5' -GGAACAGCCATGAAGGACCGC-3' and 5'-CTATAGGCCCAAAGTGCCACAAATG-3', derived from previously identified chick Syx1B (NCBI database accession number NM205074). The cDNA nucleotide sequence obtained was slightly different from that of previously identified chick Syx1B. Therefore, we registered the cloned cDNA sequence as Syntaxin 1B-2 in the DNA Data Bank of Japan database (accession number AB244720). This Syx1B was subcloned into the EcoRI site of the pEGFP-C1 vector (Clontech).

RNAi. Two pairs of Syx1B siRNA (Syx1BsiRNA1 and Syx1B-siRNA2) and scrambled siRNA (iGENE Therapeutics) were synthesized by transcription and purified by PAGE. The following siRNA sequences were used in this study: Syx1B-siRNA1, sense, $5^{\prime}$-r(GCUCCAAGUUGAAAGCCAUAGAGCAAG)- $3^{\prime}$ and antisense, 5'-r(UGCUCUAUGGCUUUCAACUUGGAGCAU)-3'; Syx1B-siRNA2, sense, $5^{\prime}$-r(CCGAUGAUAUCAAAAUGGACUCGCAAG)-3' and antisense, 5' ${ }^{\prime}$-r(UGCGAGUCCAUUUUGAUAUCAUCGGAU) $-3^{\prime}$; and scrambled siRNA, sense, $5^{\prime}$-r(CGAUUCGCUAGACCGGCUUCAUUGCAG)-3' and antisense, $5^{\prime}$-r(GCAAUGAAGCCGGUCUAGCGAAUCGAU)-3'. The RNA concentration was $40 \mathrm{~nm}$ during electroporation.

Mutagenesis. We generated an EGFP-tagged syntaxin $1 \mathrm{~B}$ with a $7 \mathrm{bp}$ same-sense mutation at the targeting region of each siRNA, which maintained the wild-type Syx1B protein sequence (SS-Syx1B). A QuikChange Multi SiteDirected Mutagenesis kit (Stratagene) was used to generate mutations. The following primers were used: $5^{\prime}$-r(GAAGACGGCCAACAAAGTGCGTTCCAAACTGAAGGCGATTGAACAGAGCATTGAGCAGGAGGAG)-3' and 5'-r(CGGGAAGTTGGCCATCTTCACGGACGACATAAAGATGGATTCTCAGATGACCAAGCAGGCCCTG)-3'.

Electroporation. In total, $7.5 \times 10^{5}$ cells were suspended in L-15 medium containing $10 \mu \mathrm{g}$ of pEGFP or pEGFP-Syx1B vector and incubated on ice for $10 \mathrm{~min}$. The cells were then transferred to chilled cuvettes (Bio-Rad) and transfected with an electroporator $(0.2 \mathrm{~V}, 950$ $\Omega$; Bio-Rad Gene Pulser). The cells were immediately suspended in $10 \mathrm{ml}$ of prewarmed L-15based culture medium, plated onto a Petri dish, incubated for $30 \mathrm{~min}$ at $37^{\circ} \mathrm{C}$ to remove cell debris, and then plated on poly-L-lysine- and laminincoated glass-bottom culture dishes. For quantification of the knockdown efficiency of Syx1B with siRNA, the cells were transfected with siRNA by electroporation. The cells were cultured with L-15-based medium and harvested by using a scraper $6 \mathrm{~h}$ after electroporation for Western blot analysis. For quantifying the effects of siRNA on macropinocytosis, growth cone collapse, and neurite outgrowth, the transfected cells were cultured in an L-15based medium supplemented with MACS Supplement B27 PLUS. In experiments involving Sema3A, the transfected cells were cultured in an F12-based medium supplemented with MACS Supplement B27 PLUS.

Imaging and quantitative analysis of fluorescence signals. For time-lapse imaging under differential interference contrast (DIC) and fluorescence microscopy, we selected growth cones that were not in contact with other cells. The growth cones were observed by using an inverted microscope (IX70; Olympus) equipped with DIC optics, GFP optics (excitation, 490 $\mathrm{nm}$; dichroic mirror, $505 \mathrm{~nm}$; emission, $515 \mathrm{~nm}$ ), DsRed optics (excitation, $555 \mathrm{~nm}$; emission, $620 \mathrm{~nm}$ ), a $60 \times$ objective (numerical aperture 1.25 ), and a xenon lamp. The images were acquired with a cooled CCD 
camera (Cool SNAP HQ; Roper Scientific) driven by MetaMorph (version 7.0) imaging software (Universal Imaging Corporation). The temperature was maintained at $37^{\circ} \mathrm{C}$ by perfusing the cells with prewarmed BSS buffer. The DIC and fluorescence images were acquired sequentially. Measurements were performed by using $2 \times 2$ binning $(1$ pixel $=0.21 \times$ $0.21 \mu \mathrm{m})$. The integrated intensity of the growth cone was quantified by subtracting the nonspecific background fluorescence.

Growth cone collapse assay. Recombinant human Sema3A/Fc chimera (Sema3A; R \& D Systems) and recombinant mouse ephrin A2/Fc chimera (ephrin A2; R \& D Systems) were used at $1 \mu \mathrm{g} / \mathrm{ml}$ for the growth cone collapse assay. Dissociated DRG neurons from embryonic day 7-9 chicks were cultured for at least $12 \mathrm{~h}$ in F-12 medium (Invitrogen) containing $50 \mathrm{ng} / \mathrm{ml} \mathrm{NGF}$. To investigate the effect of Sema3A, the culture medium was changed to F-12 medium containing $0.1 \mathrm{ng} / \mathrm{ml} \mathrm{NGF}$ at $2 \mathrm{~h}$ before the growth cone collapse assay. Subsequently, the culture medium was changed to BSS buffer. The cells were incubated for $30 \mathrm{~min}$ before their use in the growth cone collapse assay. In experiments using epoxomicin, the cells were pretreated with $1 \mu \mathrm{M}$ epoxomicin 15 min before the addition of $1 \mu \mathrm{g} / \mathrm{ml} \mathrm{Sema3A}$. Growth cone collapse was quantified as the reduction in the surface area of growth cones. The growth cone area was defined as a handlike region extending from the distal part of the neurite to the growth cone neck, whose width is equal to the width of each neurite. The width of each neurite was calculated by measuring the width of the neurite region that was $30 \mu \mathrm{m}$ from the distal part of each neurite. This calculation was adopted because the mean length of the control growth cone was $<30 \mu \mathrm{m}$. If a growth cone collapsed completely or the width of the growth cone was less than that of the each neurite, we considered the neurite region that was $30 \mu \mathrm{m}$ from the distal part of the neurite as the growth cone neck.

Macropinocytosis assay in NIH3T3 cells. NIH3T3 cells transfected with EGFP or EGFP-Syx1B were exposed to a medium containing $2.5 \mathrm{mg} / \mathrm{ml}$ of Alexa Fluor 594-dextran in the presence of serum for $30 \mathrm{~min}$. Macropinocytosis in the NIH3T3 cells was quantified by measuring the integrated fluorescence intensity of internalized Alexa Fluor 594-dextran in the cells.

Western blot analysis. The proteins were extracted in a passive lysis buffer (Promega). Then, $15 \mu \mathrm{g}$ of protein extract was subjected to $12.5 \%$ SDS-PAGE and transferred to a PVDF membrane (Millipore). The blots were blocked with $2 \%$ skimmed milk in PBS containing $0.1 \%$ Tween 20 , incubated with affinity-purified rabbit anti-Syx1B $(1 \mu \mathrm{g} / \mathrm{ml}$; Synaptic Systems) or rabbit polyclonal anti-actin antibody (1:4000 dilution; Sigma), and incubated with HRP-labeled donkey anti-rabbit IgG (Jackson ImmunoResearch). Immunoreactive bands were visualized with an Enhanced Chemiluminescence Plus detection system (GE Healthcare). For visualization of poly-ubiquitination of endogenous proteins, cells were cultured for $6 \mathrm{~h}$ with L-15-based medium and pretreated with $1 \mu \mathrm{M}$ epoxomicin 15 min before the $15 \mathrm{~min}$ Sema3A treatment. Total proteins were extracted with passive lysis buffer [10 nM ubiquitin aldehyde (Peptide Institute) and complete protease inhibitor cocktail (Roche)]. Polyubiquitinated proteins were analyzed by Western blot analysis using antiubiquitin antibody (Roche).

Immunocytochemistry. After Sema3A or vehicle treatment, the cells were fixed with $4 \%$ PFA for $1 \mathrm{~h}$ at $37^{\circ} \mathrm{C}$, followed by permeabilization with $0.3 \%$ Triton $\mathrm{X}-100$ in PBS for $2 \mathrm{~min}$ at room temperature. The cells were then immediately washed three times with a blocking solution $(1 \%$ BSA and $0.1 \%$ Triton X-100 in PBS), followed by incubation with the blocking solution for $1 \mathrm{~h}$ at room temperature and then with anti-Syx $1 \mathrm{~B}$ $(0.5 \mu \mathrm{g} / \mathrm{ml})$ and anti-synaptosomal-associated protein 25 (SNAP25) antibody (1:500 dilution, clone SMI-81; Sternberger Monoclonals) for $2 \mathrm{~h}$ at room temperature. Immunofluorescence signals were visualized by incubation with Alexa Fluor 488-labeled donkey anti-rabbit antibody (Invitrogen) and Alexa Fluor 594-labeled donkey anti-mouse antibody (Invitrogen). The integrated fluorescence intensity of Syx1B in the growth cones was normalized to that of the SNAP25 protein.

Statistical analysis. All the data are representative of at least three independent experiments. The results are expressed as the mean \pm SEM. The data were analyzed with the GraphPad Prism 4.0 program (GraphPad Software). $p<0.05$ was considered statistically significant.

\section{Results}

\section{Syx1B is a negative regulator of macropinocytosis}

To investigate the physiological role and molecular mechanism of Sema3A-induced macropinocytosis in growth cone collapse, we focused on a previous finding concerning neurotoxin $\mathrm{C} 1-$ induced phenotypes in chick DRG neurons (Igarashi et al., 1996): the total surface area of accumulated neurotoxin C1-induced vacuoles is almost equal to the expanded membrane surface area of normally growing neurites. Consequently, neurotoxin C1induced large vacuole formation was proposed to reduce the 

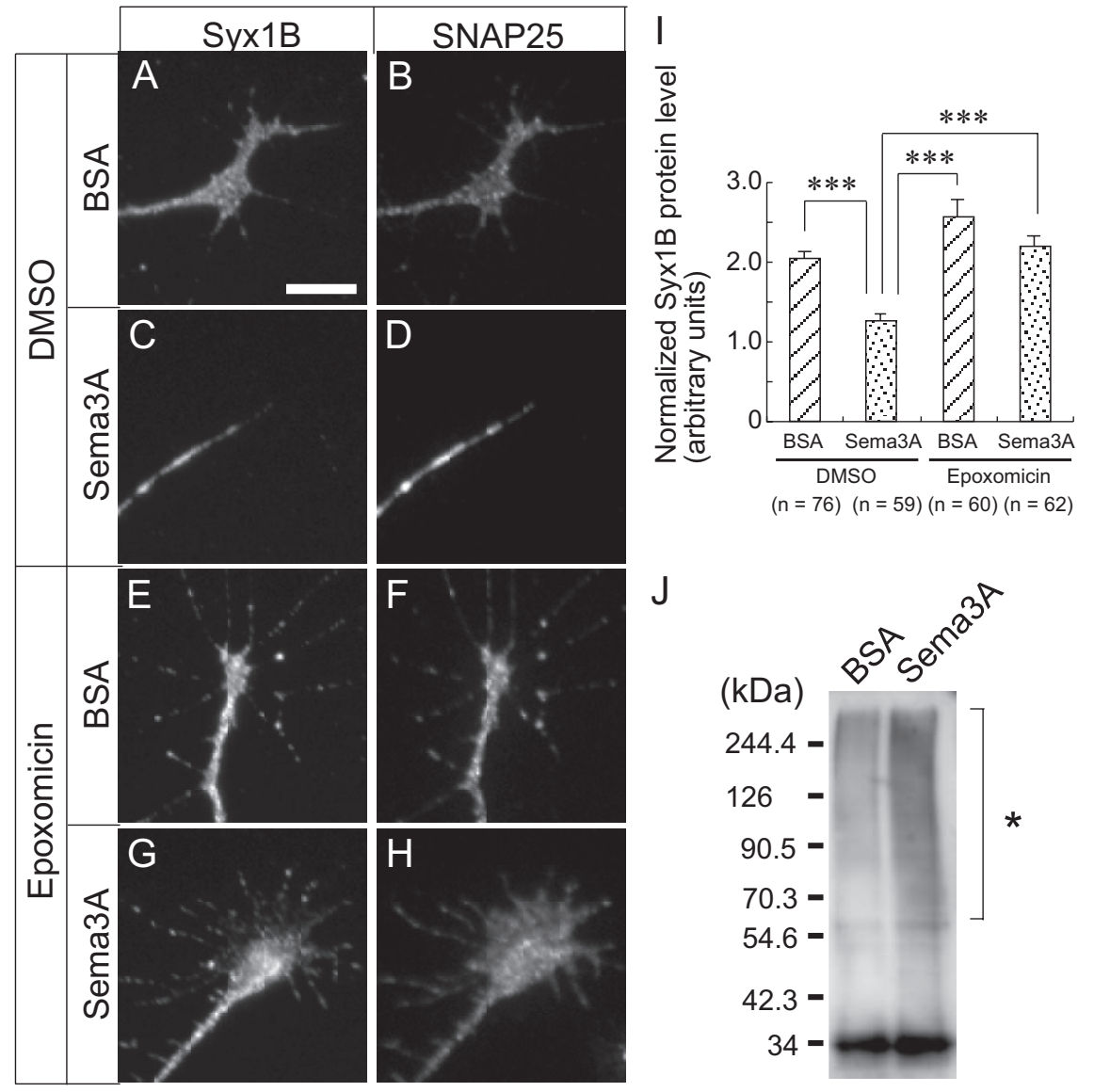

Figure 4. Sema3A decreases Syx1B protein. Immunofluorescence images of endogenous $\operatorname{Syx} 1 B(\boldsymbol{A}, \boldsymbol{C}, \boldsymbol{E}, \boldsymbol{G})$ and $\operatorname{SNAP} 25(\boldsymbol{B}, \boldsymbol{D}$, $\boldsymbol{F}, \boldsymbol{H})$ in growth cones treated with $B S A(\boldsymbol{A}, \boldsymbol{B}, \boldsymbol{E}, \boldsymbol{F})$ or Sema3A $(\boldsymbol{C}, \boldsymbol{D}, \boldsymbol{G}, \boldsymbol{H})$ for $10 \mathrm{~min}$ in the presence of DMSO $(\boldsymbol{A}-\boldsymbol{D})$ or epoxomicin $(\boldsymbol{E}-\boldsymbol{H}) . \boldsymbol{I}$, Relative amount of endogenous Syx1B protein normalized to SNAP25 in the growth cones. ${ }^{* * *} p<0.001$, two-tailed non-repeated-measures ANOVA followed by Newman-Keuls post hoc multiple comparison test. J, Poly-ubiquitination of endogenous proteins by Sema3A treatment in the presence of epoxomicin. Cell lysate was prepared from cells treated for 15 min with Sema $3 A$ or BSA in the presence of $1 \mu \mathrm{m}$ epoxomicin, immunoblotted with anti-ubiquitin antibody to detect ubiquitinated proteins as smear bands. Asterisk indicates poly-ubiquitinated proteins. Error bars indicate SEM. Scale bar, $10 \mu \mathrm{m}$.
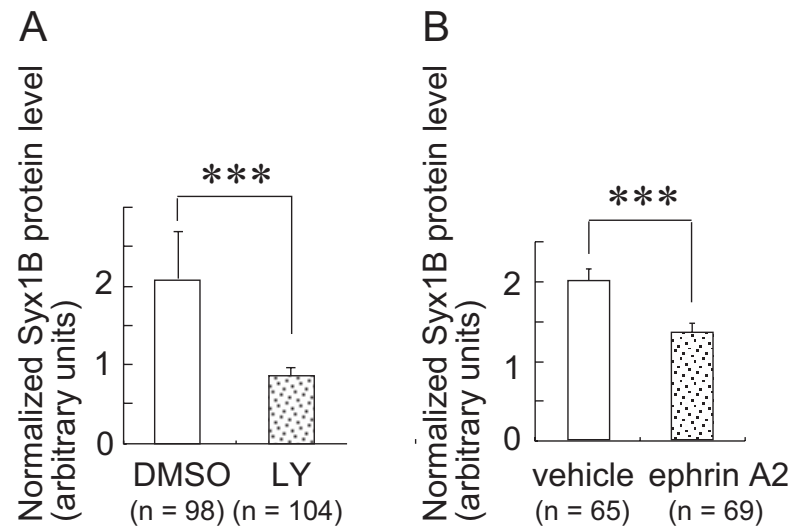

Figure 5. The Syx $1 \mathrm{~B}$ protein level in growth cones is decreased by the suppression of PI3K and ephrin $\mathrm{A} 2$ but by Jasp. $\boldsymbol{A}-\boldsymbol{C}$, Relative amount of endogenous Syx1B protein normalized to SNAP25 in the growth cones treated with LY294002 (LY, A), ephrin A2 (B), and Jasp (C). Cells were treated with the indicated drugs for $10 \mathrm{~min} .{ }^{* * *} p<0.001$, unpaired $t$ test. Error bars indicate SEM. Scale bar, $10 \mu \mathrm{m}$.

membrane supply required for neurite outgrowth (Igarashi et al., 1996). Using Alexa Fluor 488-dextran, we investigated whether neurotoxin $\mathrm{C} 1$-induced large vacuoles are formed by macropinocytosis. During 90 min neurotoxin $\mathrm{C} 1$ treatment, primary cultured DRG neurons were exposed to an Alexa Fluor 488-dextran-

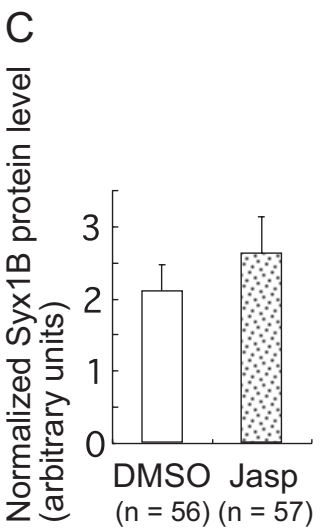

containing medium. The growth cones were imaged after washing cells with Alexa Fluor 488-dextran-deficient BSS imaging buffer. Neurotoxin C1-treated cells, not controls, exhibited marked accumulation of Alexa Fluor 488-dextran in the DIC-identifiable vacuoles of living growth cones (Fig. $1 A-F$ ). The fusion of WGA-labeled small GCVs has been postulated to form neurotoxin C1induced vacuoles (Igarashi et al., 1996). However, WGA-fluorescent puncta were not colocalized with Alexa Fluor 488dextran-positive large vacuoles in the neurotoxin C1-treated growth cones (Fig. 1G). Although neurotoxin C1-induced large vacuoles are known to move in a retrograde manner (Igarashi et al., 1996), the WGAlabeled vesicles in neurotoxin C1-treated growth cones were not transported in this manner (supplementary Movies S1, S2, available at www.jneurosci.org as supplemental material), indicating that neurotoxin $\mathrm{C} 1$-induced vacuoles were not formed by the fusion of WGA-labeled GCVs.

We next investigated whether neurotoxin C1-induced endocytosis is macropinocytosis using EIPA, a macropinocytosis-specific inhibitor. Neurotoxin C1-induced internalization of Alexa Fluor 594-dextran (Fig. $1 H, I, L, M, P)$ and reduction in growth cone surface area (Fig. $1 H, I, Q$ ) were blocked by 15 min pretreatment with $100 \mu \mathrm{M}$ EIPA (Fig. 1 J,K,N-Q). Together, these results indicate that neurotoxin $\mathrm{C} 1$-induced large vacuoles are formed by macropinocytosis and not the fusion of WGA-labeled vesicles.

Syx1B but not Syx1A is expressed in chick embryonic DRG neurons (Lü et al., 2001) and should be a target for neurotoxin C1 (Igarashi et al., 1996). We used siRNA for Syx1B (Syx1B-siRNA) to investigate whether knockdown of this molecule caused macropinocytosis, growth cone collapse, and reduced neurite outgrowth. The endogenous Syx1B level normalized to actin protein in Syx1B-siRNA-transfected DRG neurons was significantly reduced to $53.4 \pm$ $3.1 \%$ ( $p<0.0001$, two-tailed unpaired $t$ test) compared with control siRNAtransfected DRG neurons (Fig. 2A). Syx1B-siRNA-transfected cells exhibited strong accumulation of Alexa Fluor 594dextran in growth cones (Fig. 2B,F,J), growth cone collapse (Fig. $2 B, K$ ), and reduced neurite outgrowth (Fig. 2L) under physiological conditions, whereas control siRNA-transfected growth cones exhibited no such effect (Fig. 2C,G,J-L). These Syx1B knockdown-mediated phenotypes were reversed by $15 \mathrm{~min}$ pretreatment with $10 \mu \mathrm{M}$ EIPA (Fig. $2 D, H, J-L$ ).

Because siRNA-induced RNA interference in mammalian systems may have off-target effects (Jackson et al., 2003), we investigated whether Syx1B-siRNA-induced phenotypes can be 
suppressed by reintroducing Syx1B into DRG neurons. We generated an EGFPtagged syntaxin $1 \mathrm{~B}$ with a 7 bp same-sense mutation at the siRNA-targeting region, which maintained the wild-type Syx1B protein sequence (SS-Syx1B). As predicted, SS-Syx1B was expressed in growth cones cotransfected with Syx1B-siRNA (Fig. 2E). SS-Syx1B expression suppressed Syx1BsiRNA-induced uptake of Alexa Fluor 594dextran (Fig. 2I,J), growth cone collapse (Fig. $2 E, K$ ), and reduced neurite outgrowth (Fig. 2L). Furthermore, Syx1B overexpression inhibited macropinocytosis in NIH3T3 cells (supplemental Fig. S1, available at www.jneurosci.org as supplemental material). Together, these observations indicated that Syx1B is a negative regulator of macropinocytosis in the growth cones and that Syx1B-regulated macropinocytosis is required for growth cone collapse and reduced neurite outgrowth.

\section{Sema3A signaling decreases Syx1B protein level}

Previous studies demonstrated that repulsive guidance cues, including Sema3A and ephrin A2, induce endocytic vacuole formation in growth cones during growth cone collapse (Fournier et al., 2000; Jurney et al., 2002). Furthermore, we have shown that Sema3A-induced endocytic vacuoles are formed by macropinocytosis (Kabayama et al., 2009). However, the physiological role of Sema3A-induced macropinocytosis in growth cone collapse remains to be determined. We first tested the effect of EIPA on Sema3A-induced growth cone collapse. Sema3A-induced uptake of Alexa Fluor 594-dextran (Fig. $3 A, B, E, F, I)$ and growth cone collapse (Fig. $3 B, J$ ) were suppressed by $15 \mathrm{~min}$ pretreatment of cells with $10 \mu \mathrm{M}$ EIPA (Fig. 3C, D, G,H-J).

These results indicated that macropinocytosis was required for Sema3A-induced growth cone collapse and raised the possibility that Sema3A-induced macropinocytosis was mediated by the downregulation of Syx1B, because Syx1B-reduction-triggered macropinocytosis was required for growth cone collapse (Fig. 2). To examine this possibility, we tested the effect of Sema3A on Syx1B expression in growth cones. Immunocytochemistry revealed that the protein level of Syx1B in growth cones was reduced by $10 \mathrm{~min}$ Sema3A treatment (Fig. $4 A, C, I)$. The protein level of Syx1B normalized to that of SNAP25 (Fig. $4 B, D$ ) in growth cones was significantly reduced by $10 \mathrm{~min}$ Sema3A treatment (Fig. 4I). This rapid downregulation of Syx1B suggested that the protein is degraded by the proteasome. To test this, cells were exposed to a medium containing $1 \mu \mathrm{M}$ epoxomicin, a specific proteasome inhibitor (Meng et al., 1999). We found that Sema3A-induced downregulation was suppressed by epoxomicin treatment (Fig. $4 E-H, I$ ).

These results raised the possibility that Sema3A activated the ubiquitin-proteasome pathway, thereby decreasing Syx1B pro- tein. To address this issue, we performed Western blot analysis with anti-ubiquitin antibody using cell lysate prepared from cells treated with Sema3A in the presence of $1 \mu \mathrm{M}$ epoxomicin. Western blot analysis with anti-ubiquitin antibody demonstrated that a broad band with high molecular weight was increased in response to Sema3A in the presence of epoxomicin (Fig. $4 J$ ). These results indicate that Sema3A activates the ubiquitin-proteasome pathway.

Suppression of the basal activity of phosphatidylinositol 3-kinase (PI3K) is known to be required for Sema3A-induced growth cone collapse and neurite retraction (Chadborn et al., 2006; Orlova et al., 2007). Interestingly, PI3K is also known as a mediator of macropinocytosis (Swanson and Watts, 1995). Therefore, we next tested the effect of PI3K inhibition on Syx1B protein expression using the PI3K inhibitor LY294002 [2-(4-morpholinyl)8-phenyl-1(4H)-benzopyran-4-one]. The protein level of Syx1B normalized to that of SNAP25 in the growth cones was significantly reduced by $10 \mathrm{~min}$ LY294002 treatment (Fig. 5A). 

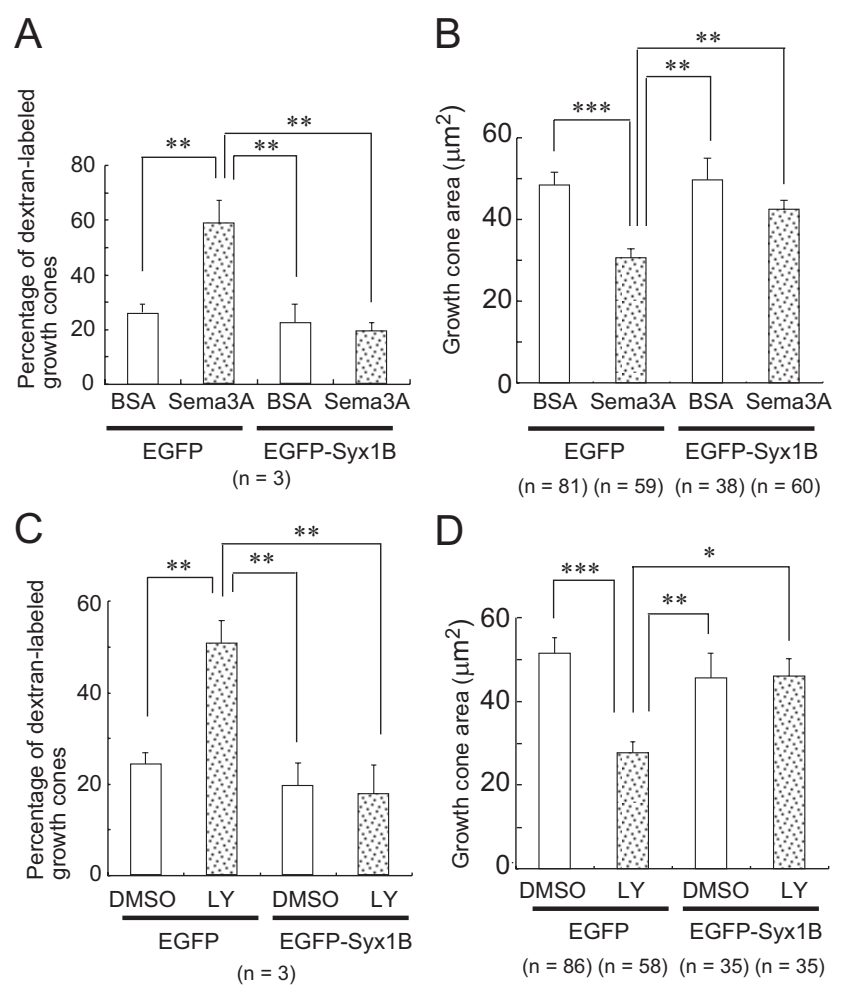

Figure 7. Overexpression of EGFP-Syx1B suppresses Sema3A- or LY294002 (LY)-induced macropinocytosis and growth cone collapse. The percentage of growth cones labeled with Alexa Fluor 594 - dextran $(\boldsymbol{A}, \boldsymbol{C})$ and growth cone area $(\boldsymbol{B}, \boldsymbol{D})$ of EGFP- or EGFP-Syx1B-transfected growth cones subjected to 10 min treatment with BSA, Sema3A, DMSO, or LY294002 in the presence of Alexa Fluor 594-dextran. ${ }^{*} p<0.05,{ }^{* *} p<0.01,{ }^{* * *} p<0.001$, two-tailed non-repeated-measures ANOVA followed by Newman-Keuls post hoc multiple comparison test. Error bars indicate SEM.

We next examined the effect of ephrin A2, a repulsive guidance cue, on Syx1B expression because ephrin A2 induces large endocytic vacuoles during growth cone collapse (Jurney et al., 2002). Expression of Syx1B normalized to SNAP25 was decreased by 10 min treatment with ephrin A2 (Fig. $5 B$ ). In contrast, expression of Syx1B normalized to SNAP25 was not decreased in growth cones treated with jasplakinolide (Jasp), an inhibitor of F-actin depolymerization that induces growth cone collapse-like morphological changes (Kabayama et al., 2009) (Fig. 5C). These results indicate that reduction of Syx1B protein levels is not secondary to the morphological changes in growth cones during growth cone collapse. Together, these results suggest that Sema3A-PI3K signaling decreases Syx1B protein in growth cones, thereby inducing macropinocytosis.

\section{Syx1B couples macropinocytosis to Sema3A-induced growth cone collapse}

We next performed a gain-of-function analysis by maintaining Syx1B expression at high levels after Sema3A treatment in the overexpression experiment (supplemental Fig. S2, available at www.jneurosci.org as supplemental material). The overexpression of EGFP-Syx1B reduced Sema3A- or LY294002-induced macropinocytosis in the growth cones (Figs. 6A-D, I-L, $7 A, C$ ) and reversed the Sema3A- or LY294002-induced reduction in growth cone area (Figs. $6 A-D, I-L, 7 B, D$ ), whereas the overexpression of EGFP alone had no such effect (Figs. $6 E-H, M-P$, $7 A-D)$. These observations indicated that macropinocytosis triggered by Syx1B reduction plays a positive role in Sema3Adependent growth cone collapse.

\section{Discussion}

Macropinosomes can be visualized by the incorporation of highmolecular-weight $(>10 \mathrm{kDa})$ dextran and are reportedly formed independently by clathrin-mediated endocytosis (Swanson and Watts, 1995). We demonstrated for the first time that neurotoxin C1-induced large vacuoles are formed by macropinocytosis (Fig. 1). Because the total surface area of accumulated neurotoxin $\mathrm{C} 1$ induced vacuoles is almost equal to the expanded membrane surface area of normally growing neurites (Igarashi et al., 1996), we concluded that macropinocytosis-mediated retrieval of the plasma membrane contributes to neurotoxin $\mathrm{C} 1$-induced reduction in growth cone area, which is supported by the finding that macropinocytosis-specific inhibitor EIPA suppressed neurotoxin $\mathrm{C} 1$-induced reduction in growth cone area and dextran uptake (Fig. 1).

Syx1B is a substrate for neurotoxin C1 (Igarashi et al., 1996). We found that knockdown of Syx1B with siRNA was sufficient for the induction of macropinocytosis and growth cone collapse (Fig. 2). Because EIPA suppressed Syx1B-siRNA-induced uptake of dextran and growth cone collapse (Fig. 2), we concluded that Syx1B is a negative regulator of macropinocytosis required for growth cone collapse. In addition, these results suggested that basal levels of Syx1B protein were sufficient to suppress macropinocytosis during basal neurite outgrowth, which is consistent with the findings that the percentage of Alexa Fluor 594dextran-labeled growth cones was not significantly reduced by EIPA treatment in the absence of Sema3A (Fig. 3) and that Syx1B overexpression does not affect basal neurite outgrowth in the absence of Sema3A (data not shown).

The molecular mechanisms by which Syx1B suppresses macropinocytosis are unclear at present. Macropinocytosis has been observed in non-neuronal cells such as NIH3T3 cells in the presence of serum (Dharmawardhane et al., 2000), suggesting the existence of common molecular machinery for macropinocytosis in neurons and non-neuronal cells. Syx1B may suppress this common mechanism of macropinocytosis, because Syx1B overexpression also inhibited macropinocytosis in NIH3T3 cells in the presence of serum (supplemental Fig. S1, available at www. jneurosci.org as supplemental material). The small GTPase Rac1 is also involved in growth cone collapse (Jin and Strittmatter, 1997) and required for Sema3A-induced endocytic vacuole formation (Jurney et al., 2002). Racl (West et al., 2000) and its downstream effecter Pak1 (Dharmawardhane et al., 2000) induce macropinocytosis by actin reorganization without affecting clathrin-mediated endocytosis. Furthermore, a recent study revealed that amiloride, a specific inhibitor of macropinocytosis, inhibits macropinocytosis by preventing Racl and cdc42 signaling (Koivusalo et al., 2010). These reports raise the possibility that a reduction in Syx1B levels may activate a Rac1-dependent macropinocytic pathway. G-protein-coupled receptor kinaseinteracting target (Git1), which is involved in neurite growth of retinal neurons (Za et al., 2006), also activates Pak1 (Zhao et al., 2000; Loo et al., 2004). Interestingly, we found that Git1 overexpression induced macropinocytosis and that Syx1B overexpression suppressed Git1-induced macropinocytosis in NIH3T3 cells (data not shown). Therefore, whether a reduction in Syx1B levels activates pathways involved in small GTPase- or related proteindependent macropinocytosis in growth cones is a topic of interest for future research.

In a previous study, we demonstrated a correlation between the reduction in growth cone area and the area of Sema3Ainduced macropinosomes (Kabayama et al., 2009), suggesting a 
positive role for macropinocytosis in Sema3A-induced growth cone collapse. In the present study, we have shown for the first time that EIPA suppresses Sema3A-induced growth cone collapse (Fig. 3), indicating that macropinocytosis has a positive role in Sema3A-induced growth cone collapse. Sema3A treatment rapidly decreased the Syx1B protein level in growth cones (Fig. 4), which indicates that Sema3A signaling regulates Syx1B expression and is supported by the finding that suppression of PI3K, a downstream mediator of Sema3A, also caused a rapid reduction in Syx1B protein levels (Fig. 5). The Sema3A-induced rapid reduction of Syx1B was suppressed by the epoxomicin treatment (Fig. 4). Furthermore, we have shown for the first time that Sema3A activates the ubiquitin-proteasome pathway (Fig. 4). These results raise the possibility that Sema3A decreases Syx1B protein by activating the ubiquitin-proteasome pathway. Additional studies are required to assess this possibility.

Because Syx1B is a negative regulator of macropinocytosis (Figs. 1, 2), we concluded that Sema3A-induced macropinocytosis was dependent on a reduction in the Syx1B protein level, because Sema3A- or LY294002-induced macropinocytosis was suppressed by Syx1B overexpression (Figs. 6, 7). Furthermore, suppression of macropinocytosis by Syx1B overexpression inhibited the Sema3A- or LY294002-induced reduction in growth cone area (Figs. 6, 7). These results indicate that Syx1B-reduction-triggered macropinocytosis is required for Sema3A-induced growth cone collapse. This mechanism might be common for repulsive guidance-cue-mediated growth cone collapse, because ephrin A2 also reduces Syx1B protein level in growth cones. Additional studies focusing on Syx1B-regulated macropinocytosis may accelerate our understanding of the molecular mechanisms of repulsive axon guidance.

In summary, macropinocytosis-mediated membrane retrieval is a critical process for Sema3A-induced growth cone collapse and axon retraction, which entail a reduction in large areas of the plasma membrane. When macropinocytosis-mediated retrograde membrane trafficking is suppressed, axons can elongate. This is the first study to reveal the role of macropinocytosis in Sema3A-induced axon growth inhibition.

\section{References}

Chadborn NH, Ahmed AI, Holt MR, Prinjha R, Dunn GA, Jones GE, Eickholt BJ (2006) PTEN couples Sema3A signalling to growth cone collapse. J Cell Sci 119:951-957.

Dharmawardhane S, Schürmann A, Sells MA, Chernoff J, Schmid SL, Bokoch GM (2000) Regulation of macropinocytosis by p21-activated kinase-1. Mol Biol Cell 11:3341-3352.

Engqvist-Goldstein AE, Drubin DG (2003) Actin assembly and endocytosis: from yeast to mammals. Annu Rev Cell Dev Biol 19:287-332.

Fournier AE, Nakamura F, Kawamoto S, Goshima Y, Kalb RG, Strittmatter SM (2000) Semaphorin3A enhances endocytosis at sites of receptor-Factin colocalization during growth cone collapse. J Cell Biol 149:411-422.

Igarashi M, Kozaki S, Terakawa S, Kawano S, Ide C, Komiya Y (1996) Growth cone collapse and inhibition of neurite growth by Botulinum neurotoxin C1: a t-SNARE is involved in axonal growth. J Cell Biol 134:205-215.

Jackson AL, Bartz SR, Schelter J, Kobayashi SV, Burchard J, Mao M, Li B, Cavet G, Linsley PS (2003) Expression profiling reveals off-target gene regulation by RNAi. Nat Biotechnol 21:635-637.

Jin Z, Strittmatter SM (1997) Rac1 mediates collapsin-1-induced growth cone collapse. J Neurosci 17:6256-6263.

Johannes L, Lamaze C (2002) Clathrin-dependent or not: is it still the question? Traffic (Copenhagen) 3:443-451.
Jurney WM, Gallo G, Letourneau PC, McLoon SC (2002) Rac1-mediated endocytosis during ephrin-A2- and semaphorin 3A-induced growth cone collapse. J Neurosci 22:6019-6028.

Kabayama H, Takei K, Fukuda M, Ibata K, Mikoshiba K (1999) Functional involvement of synaptotagmin I/II C2A domain in neurite outgrowth of chick dorsal root ganglion neuron. Neuroscience 88:999-1003.

Kabayama H, Nakamura T, Takeuchi M, Iwasaki H, Taniguchi M, Tokushige N, Mikoshiba K (2009) $\mathrm{Ca}^{2+}$ induces macropinocytosis via F-actin depolymerization during growth cone collapse. Mol Cell Neurosci 40:27-38.

Koivusalo M, Welch C, Hayashi H, Scott CC, Kim M, Alexander T, Touret N, Hahn KM, Grinstein S (2010) Amiloride inhibits macropinocytosis by lowering submembranous $\mathrm{pH}$ and preventing $\mathrm{Racl}$ and $\mathrm{Cdc} 42$ signaling. J Cell Biol 188:547-563.

Kurazono H, Shimozawa K, Hosokawa M, Sakaguchi G (1985) Procedures for large-scale production and purification of clostridium-botulinum C1toxin for preparation of toxoid. Fems Microbiol Lett 30:47-51.

Loo TH, Ng YW, Lim L, Manser E (2004) GITI activates p21-activated kinase through a mechanism independent of p21 binding. Mol Cell Biol 24:3849-3859.

Lü Q, AtKisson MS, Jarvis SE, Feng ZP, Zamponi GW, Dunlap K (2001) Syntaxin 1A supports voltage-dependent inhibition of $\alpha 1 \mathrm{~B} \mathrm{Ca}^{2+}$ channels by $\mathrm{G} \beta \gamma$ in chick sensory neurons. J Neurosci 21:2949-2957.

Luo Y, Raible D, Raper JA (1993) Collapsin: a protein in brain that induces the collapse and paralysis of neuronal growth cones. Cell 75:217-227.

Meng L, Mohan R, Kwok BH, Elofsson M, Sin N, Crews CM (1999) Epoxomicin, a potent and selective proteasome inhibitor, exhibits in vivo antiinflammatory activity. Proc Natl Acad Sci U S A 96:10403-10408.

Mercer J, Helenius A (2009) Virus entry by macropinocytosis. Nat Cell Biol 11:510-520.

Mikule K, Gatlin JC, de la Houssaye BA, Pfenninger KH (2002) Growth cone collapse induced by semaphorin $3 \mathrm{~A}$ requires 12/15-lipoxygenase. J Neurosci 22:4932-4941.

Orlova I, Silver L, Gallo G (2007) Regulation of actomyosin contractility by PI3K in sensory axons. Dev Neurobiol 67:1843-1851.

Piper M, Salih S, Weinl C, Holt CE, Harris WA (2005) Endocytosisdependent desensitization and protein synthesis-dependent resensitization in retinal growth cone adaptation. Nat Neurosci 8:179-186.

Püschel AW, Adams RH, Betz H (1995) Murine semaphorin D/collapsin is a member of a diverse gene family and creates domains inhibitory for axonal extension. Neuron 14:941-948.

Schiavo G, Shone CC, Bennett MK, Scheller RH, Montecucco C (1995) Botulinum neurotoxin type $\mathrm{C}$ cleaves a single Lys-Ala bond within the carboxyl-terminal region of syntaxins. J Biol Chem 270:10566-10570.

Swanson JA, Watts C (1995) Macropinocytosis. Trends Cell Biol 5:424-428.

Taniguchi M, Shimizu T (2004) Characterization of a novel member of murine semaphorin family. Biochem Biophys Res Commun 314:242-248.

Taniguchi M, Yuasa S, Fujisawa H, Naruse I, Saga S, Mishina M, Yagi T (1997) Disruption of semaphorin III/D gene causes severe abnormality in peripheral nerve projection. Neuron 19:519-530.

Taniguchi M, Nagao H, Takahashi YK, Yamaguchi M, Mitsui S, Yagi T, Mori K, Shimizu T (2003) Distorted odor maps in the olfactory bulb of semaphorin 3A-deficient mice. J Neurosci 23:1390-1397.

Wadia JS, Stan RV, Dowdy SF (2004) Transducible TAT-HA fusogenic peptide enhances escape of TAT-fusion proteins after lipid raft macropinocytosis. Nat Med 10:310-315.

Wadia JS, Schaller M, Williamson RA, Dowdy SF (2008) Pathologic prion protein infects cells by lipid-raft dependent macropinocytosis. PLoS One 3:e3314

West MA, Prescott AR, Eskelinen EL, Ridley AJ, Watts C (2000) Rac is required for constitutive macropinocytosis by dendritic cells but does not control its downregulation. Curr Biol 10:839-848.

Za L, Albertinazzi C, Paris S, Gagliani M, Tacchetti C, de Curtis I (2006) beta PIX controls cell motility and neurite extension by regulating the distribution of GIT1. J Cell Sci 119:2654-2666.

Zhao ZS, Manser E, Loo TH, Lim L (2000) Coupling of PAK-interacting exchange factor PIX to GIT1 promotes focal complex disassembly. Mol Cell Biol 20:6354-6363. 